\title{
Object-Oriented Programming Course Reform Using Python Language in the Background of Artificial Intelligence
}

\author{
Yongcheng $\mathrm{Wu}$ \\ Computer Engineering School \\ Jingchu University of Technology \\ Jingmen, China \\ wuyongcheng11@126.com
}

\begin{abstract}
Using C++ or Java as the describing language is the traditional way of teaching of object-oriented programming (OOP). Currently with the rapid development of AI and Python programming language, teaching OOP using Python become more and more important and suitable. In this paper, firstly the disadvantages of $\mathrm{C}++$ and Java languages as well as the advantages of Python for OOP teaching are discussed. Then the main content of OOP teaching using Python is put forward, which consists of two parts: the basic part and application part. The basic part should include the basic grammar of Python and basic knowledge and skills of OOP. For the application part, we suggest using an AI library Sklearn to practice some practical AI application. A specific instance of $\mathrm{K}$-means clustering case teaching using Sklearn is also discussed in this paper.
\end{abstract}

Keywords-course reform; object-oriented programming; Python language; artificial intelligence

\section{INTRODUCTION}

The course of object-oriented Programming is a compulsory professional basic course for the students majoring in computer science and technology. It is also an important elective for students not majoring in computer science. The teaching objective of the course is to teach students to master the ideas and design methods of objectoriented programming, and cultivate students basic ability to apply object-oriented thinking to handle and solve practical problems [1].

The course of object-oriented programming mainly consists of the basic ideas of OOP (object-oriented programming), such as classes and objects, overloading, encapsulation, inheritance, virtual functions, polymorphism, etc. These issues are more abstract and difficult for beginners to understand. At the same time, it is a highly practical course, which means we should use an object-oriented programming language as a descriptive tool, such as $\mathrm{C}++$ or JAVA, to elaborate the main concepts, design patterns and programming methods of OOP. Only by using and practicing these kinds of specific programming languages can students really master the abstract knowledge of OOP they have learned in class, which shows that the programming language for teaching of OOP is very critical.
With the rapid development of artificial intelligence (AI) our society is being greatly changed. In 2017, China issued a landmark AI development plan, New Generation AI Development Planning, which provides strategic support for the development of AI in China [2]. In 2018, China's Ministry of Education formulated the Action Plan for Artificial Intelligence Innovation in Colleges [3].

Currently, Python has become the most widely used AI programming language all over the world [4]. On the one hand, it provides strong support for the application of AI. On the other hand, it is regarded as the closest programming language to human natural language, which facilitates people to study and use. In order to make the developers focus on business logic rather than grammar details, Python pays much attention to the readability and conciseness of the program, abstracts a lot of contents, and can complete more powerful functions by using as little code as possible. It can not only cultivate students' interest in learning OOP, but also improve students' application ability and practical project ability.

\section{ThE ADVANTAgES OF OOP UsING PYTHON}

\section{A. The Disadvantages of Traditional OOP Teaching}

In the past decade, many colleges in China, have adopted $\mathrm{C}++$ or JAVA as the programming language in the teaching of object-oriented programming for computer science and technology and other specialties. In most of the teaching textbooks of OOP, it always takes two to three chapters to expound basic methods and techniques of OOP, the basic knowledge of $\mathrm{C}++$ or JAVA language and its development environment, so that students can lay a foundation for the study of the following chapters. There are several reasons for using $\mathrm{C}++$ or JAVA as the teaching language of OOP course. The core reason is that $\mathrm{C}++$ and JAVA are typical objectoriented programming languages, which conform to the basic features of OOP, such as class, object, encapsulation, inheritance and polymorphism.

In the past years, the author used $\mathrm{C}++$ or JAVA as the teaching language in the course of OOP. Various teaching methods such as case-study, discussion-method, project-driven have been applied. However, in terms of teaching effect, it is 
not ideal. The number of students who achieve excellent results is less, the number of people in medium and general grade is the largest. Through analyzing the distribution and communicating with students, it reveals that the students who have excellent learning results basically have a good grasp of $\mathrm{C}++$ or JAVA language, strong programming practical ability, and some of them already have experience of participating in software development. For the students of medium and general grade, the main cause is that they have not mastered the grammar of $\mathrm{C}++$ or JAVA well enough. They could not even write a complete program in these two languages, which makes it more difficult for them to understand and apply the abstract and complex concepts in OOP.

The reason for this is that due to the complexity of $\mathrm{C}++$ or JAVA language itself and its development environment, studying the course of OOP becomes more difficult. Teachers often have to spend a lot of time teaching $\mathrm{C}++$ or JAVA language grammar knowledge to students, and students have to spend a lot of time and energy to grasp the basic knowledge of the language and program developing environment. In the case of limited class hours, object-oriented programming courses often turn into $\mathrm{C}++$ or JAVA language learning courses. Especially during the practice class, students often spend a lot of time debugging because of some grammar errors, which might make students neglect or reduce more important content of OOP itself such as OOP paradigm, design pattern, polymorphism, etc [5].

\section{B. The Advantages of OOP Using Python}

Python is an object-oriented, interpretative, dynamic data class, high-level programming language with concise and clear grammar and rich and powerful class libraries. Since Python language is simple, readable and extensible, some famous universities abroad have offered programming course using Python as the describing language. The course of Introduction to Computer Science and Programming at MIT is taught in Python. The following features of Python are very conducive to the teaching of OOP.

- Python adheres to a clear and uniform style in design. Its code is easy to read, to learn and maintain. Reading a good Python program is like reading English. It enables students to concentrate on object-oriented programming rather than understanding the language itself. Therefore, Python language has great advantages in learning object-oriented programming.

- Python is a fully object-oriented language, in which functions, modules, strings are all objects. It fully supports encapsulation, inheritance, overloading, and polymorphism. Its content can meet the requirement of OOP teaching [6].

- The execution of Python language is based on virtual machine mechanism. And its virtual machine is highly abstract, which makes students need not have too much knowledge about computer hardware. It is also a highlevel language, without considering the underlying details such as memory management, making it easy for students not majoring in computer to use it to write programs, and the standard code requirements make it more readable. If the code of Python violate the indentation rules, the program cannot be compiled, which is very crucial to force students to establish good programming habits.

- Python can run in interactive mode and can be run directly in command mode in mainstream operating systems. This feature is very suitable for Python to be used in classroom teaching whose total class hours are limited.

It has abundant standard libraries and extended libraries, such as network communication, graphical user interface, database and scientific computing. Python is a free and opensource, and it's easy to get the latest releases and libraries. Python program can be written directly in the interactive environment or programmed in a graphical integrated development environment.

\section{COURSE REFORM FOR OOP USING PYTHON LANGUAGE}

In order to teach the course of OOP Using Python Language more productively, the course reform can be conducted as follows.

\section{A. Determining Main Teaching Contents}

When selecting teaching contents of OOP Using Python, since Python language is easy to learn and use, more time can be allocated to more important object-oriented programming issues. The choosing of teaching contents does not require students to have too much programming foundation and can be flexibly adjusted according to the students' foundation. Table 1 lists the teaching contents and class hours allocation of the course of OOP Using Python.

TABLE I. TEACHING CONTENT AND TIME DistRIBUTION

\begin{tabular}{|c|c|c|}
\hline \multicolumn{2}{|c|}{ Teaching Contents } & $\begin{array}{c}\text { Teaching Time } \\
\text { Distribution } \\
\text { (class hours) }\end{array}$ \\
\hline \multirow{4}{*}{ Basic part } & Introduction to Python & 8 \\
\cline { 2 - 3 } & Class and object & 8 \\
\cline { 2 - 3 } & Inheritance & 4 \\
\cline { 2 - 3 } & Overloading & 4 \\
\cline { 2 - 3 } & Polymorphism & 6 \\
\cline { 2 - 3 } & Template & 4 \\
\cline { 2 - 3 } & Exception handling & 2 \\
\hline Application & File operation and & 4 \\
\hline part & serialization & 8 \\
\hline \multicolumn{2}{|c|}{ Python+sklearn } & 48 \\
\hline
\end{tabular}

The teaching contents of OOP using Python can be divided into two parts: the basic part and the application part.

The basic part mainly includes: Python basic grammar rules, and Python object-oriented programming foundation.

The application part is to cultivate students' ability to solve practical problems using what they have learned in the basic part of OOP, to promote students' interest in learning program design. Since Python is the most important AI developing language, in the application part, we choose Python+sklearn 
for teaching. Sklearn is a basic and significant machine learning library. It provides and encapsulates many machine learning algorithms, such as classification, clustering, regression, etc. By learning Python+sklearn, we can not only practice to deal with practical problems using Python, but also accelerate the popularization and application of AI technology, which is critical in the background of AI.

\section{B. AI Case Teaching of Application Part of OOP}

According to the characteristics of OOP using Python, we should encourage students to develop some small projects to increase their practical ability. There are many existing textbooks introduce basic grammar and programming structure of Python. On the contrary, the application of AI methods is relatively complex and abstruce, which would make students feel difficult to understand.

In Table 1, the class hours allocate to application part are 8 In order to make students master more knowledge and skills in the shorter experimental class hours, it is necessary to carefully design the experimental projects of Python+sklearn. At the same time, it is suggested to increase the practical class hours and introduce cases in practical teaching to help students enhance their understanding of artificial intelligence algorithms. Through case teaching, machine learning method can be further extended to practical application.

Clustering is a very common and important machine learning scheme. In practical application, taking e-commerce as an example, we can identify customers with similar browsing behavior by clustering, and then divide them into different customer groups. It can be used as the basis for analyzing the common features of similar customers, depicting the purchase mode of customer groups, so as to help ecommerce platform understand customers better and provide them more suitable services.

For the course of OOP using Python, K-means is a very simple clustering algorithm [7]. Its principle is very clear and can be used as teaching cases.

K-means divides a set of $\mathrm{N}$ data objects into k parts $(\mathrm{k} \leqslant \mathrm{n})$ where each part represents a cluster. The algorithm description is shown in Fig. 1.

Each customer's feature data can be regarded as a data point in space. For simplicity, we can quickly generate sample points in two-dimensional space through sklearn to form data sets for clustering analysis. We can call make_moons method to generate crescent-shaped discrete data, and call make_blobs method to generate blob-shaped discrete data. It is easy to understand from the name of the method, which shows that Python standard library method is intuitive and good reading.
Input: $\mathrm{k}$ (cluster number), dataset $\mathrm{S}$ (with $\mathrm{n}$ instances)

Output: clusters

Process:

1. choose $\mathrm{n}$ instances from $\mathrm{S}$ as the original center of clustering;

2. repeat the step 3 and 4 until every cluster does not change;

3. According to the mean value of each cluster (center), the distance between each instance and these center is calculated, and re-divided $\mathrm{S}$ according to the minimum distance;

4. If a cluster changes, then calculate its mean again.

Fig. 1. Algorithm of K-means

The program of how to build a dataset is shown in Fig 2 .

from sklearn import datasets

$\mathrm{X} 1, \mathrm{y} 1=$ datasets.make_moons(n_samples $=3000$, noise $=.05$ )

X2, y2 =datasets.make_blobs(n_samples $=1000$,

n_features $=2$, centers $=[[1.2,1.2]]$,

cluster_std=[[.1]])

Fig. 2. Using Python to produce datasets

where $n \_$sample is the number of samples to be produced; noise represents the degree of scattering of sample points, the larger the value, the more scattered; $n$ features is the feature number of each sample; centers represent the center of sample points; cluster_std represents the standard deviation of each cluster.

Fig. 3 illustrates how to use K-means method to cluster the dataset.

from sklearn.cluster import KMeans

$\mathrm{y}=$ KMeans(n_clusters $=3$, max_iter $=10000$, tol=1e-3, random_state=9).fit_predict(X)

Fig. 3. Using Python to cluster dataset with K-means

In Fig. 3, by importing the K-means class in sklearn.cluster, the object is created and the fit_predict( ) method is called to cluster the dataset. The program is simple and easy to understand. There are some commonly used parameters for creating K-means objects: $n \_$clusters denotes the number of clusters produced by K-means method. random_state represents the seeds of a random generator and is related to the initialization center. max_iter denotes the 
maximum number of iteration. tol is the least tolerant deviation. When the deviation is less than tol, the program exits the iteration.

By introducing case teaching and integrating it into OOP teaching, students can intuitively learn and master different machine learning methods, and then expand to a wider field of artificial intelligence. Artificial intelligence talent training is a long-term process, which needs to penetrate into all aspects of teaching. We need to reform teaching methods, change educational ideas to train talents, and promote the development of AI technology.

\section{Assessment Model Reform}

The purpose of the assessment model reform is to spur students to attach more importance to application and practice. The weighting of "comprehensive design assignment" should be increased. The final score consists of normal performance (20\%), comprehensive design assignment (40\%) and final written examination (40\%).

\section{CONCLUSION}

In this paper, we analyze the disadvantages of the traditional teaching of OOP, the advantages of OOP teaching using Python, the necessity and urgency of offering the course of object-oriented programming using Python. We also propose that the course content of OOP using Python should contain basic part and application part. For the application part, due to the significance of AI and its close relationship with Python, we choose a popular AI library sklearn to cultivate students' practical programming ability. Also, a case teaching of sklearn about K-means clustering is elaborated in this paper.
The theory of OOP is integrated into the actual hot topics of AI and students' learning enthusiasm is to be improved.

At present, there are few textbooks introducing objectoriented programming in Python language. Most textbooks are expounding Python language itself. They are not suitable for the course of object-oriented programming. Therefore, corresponding textbooks need to be constructed. In addition, the construction of teaching resources adapted to the course content is also a problem to be solved.

\section{REFERENCES}

[1] Z. Ding, J. Qian, “Teaching innovation of object-oriented programming course”, in Computer Education, 2011(9): 9. (In Chinese)

[2] New Generation Development Planning of Artificial Intelligence [Z]. Central Government file GuoFa [2017] 35. http://www.gov.cn/zhengce/content/201707/20/content_5211996.htm. (In Chinese)

[3] Action Plan for Artificial Intelligence Innovation in Colleges. Ministry of Education file JiaoJi [2018] 3. http://www.cac.gov.cn/201804/11/c_1122663790.htm. (In Chinese)

[4] D. Wei, X. Wang, Y. Wang, Y, Chen, S. Wang, "Construction and Teaching Practice of Programming General Course Based on Python”, in Computer Education, 2019(2): 69-73. (In Chinese)

[5] Y. Bai, "Research on the teaching of Python language as an introductory programming language”, in Microcomputer Application, 2019 (35):1. (In Chinese)

[6] T. Song, T. Huang, X. Li, "Python Language: Ideal Choice for Teaching Reform of Programming Course”, in China University Teaching, 2016(2): 42-47. (In Chinese)

[7] L. Wang, Z. Hao, "Case Teaching Research of K-means Clustering Algorithm”, in Computer Education, 2016(8):152-157. (In Chinese) 